\title{
Resistance to FGFR1-targeted therapy leads to autophagy via TAK1/ AMPK activation in gastric cancer
}

\author{
Rui Peng ${ }^{1} \cdot$ Yan Chen ${ }^{2,5} \cdot{\text { Liangnian } \mathrm{Wei}^{3} \cdot \mathrm{Gang} \mathrm{Li}^{1} \cdot \text { Dongju Feng }^{3} \cdot \text { Siru Liu }^{2} \cdot \text { Runqiu Jiang }^{4} \cdot \text { Shaojiang Zheng }}^{2}$. \\ Yun Chen ${ }^{1,3,6}$
}

Received: 9 January 2020 / Accepted: 15 May 2020 / Published online: 2 July 2020

(c) The International Gastric Cancer Association and The Japanese Gastric Cancer Association 2020

\begin{abstract}
Background Fibroblast growth factor receptor 1 (FGFR1) is frequently dysregulated in various tumors. FGFR inhibitors have shown promising therapeutic value in several preclinical models. However, tumors resistant to FGFR inhibitors have emerged, compromising therapeutic outcomes by demonstrating markedly aggressive metastatic progression; however, the underlying signaling mechanism of resistance remains unknown.

Methods We established FGFR inhibitor-resistant cell models using two gastric cancer (GC) cell lines, MGC-803 and BGC823. RNA-seq was performed to determine the continuous cellular transcriptome changes between parental and resistant cells. We explored the mechanism of resistance to FGFR inhibitor, using a subcutaneous tumor model and GC patient-derived tumor organotypic culture.

Results We observed that FGFR1 was highly expressed in GC and FGFR1 inhibitor-resistant cell lines, demonstrating elevated levels of autophagic activity. These resistant cells were characterized by epithelial-mesenchymal transition (EMT) required to facilitate metastatic outgrowth. In drug-resistant cells, the FGFR1 inhibitor regulated GC cell autophagy via AMPK/mTOR signal activation, which could be blocked using either pharmacological inhibitors or essential gene knockdown. Furthermore, TGF- $\beta$-activated kinase 1 (TAK1) amplification and metabolic restrictions led to AMPK pathway activation and autophagy. In vitro and in vivo results demonstrated that the FGFR inhibitor AZD4547 and TAK1 inhibitor NG25 synergistically inhibited proliferation and autophagy in AZD4547-resistant cell lines and patient-derived GC organotypic cultures.

Conclusions We elucidated the molecular mechanisms underlying primary resistance to FGFR1 inhibitors in GC, and revealed that the inhibition of FGFR1 and TAK1 signaling could present a potential novel therapeutic strategy for FGFR1 inhibitor-resistant GC patients.
\end{abstract}

Keywords Gastric cancer · FGFR1 · TAK1 · Autophagy · Drug resistance

\begin{tabular}{l} 
Rui Peng, Yan Chen and Liangnian Wei contributed equally to this \\
work. \\
\hline Electronic supplementary material The online version of this \\
article (https://doi.org/10.1007/s10120-020-01088-y) contains \\
supplementary material, which is available to authorized users. \\
\hline $\begin{array}{l}\text { Runqiu Jiang } \\
\text { jiangrq@ njmu.edu.cn }\end{array}$ \\
$\begin{array}{l}\text { Shaojiang Zheng } \\
\text { zhenghoho@aliyun.com }\end{array}$ \\
$\begin{array}{l}\text { Yun Chen } \\
\text { chenyun @ njmu.edu.cn }\end{array}$ \\
Extended author information available on the last page of the article
\end{tabular}

$\begin{array}{ll}\text { Abbreviations } \\ \text { FGFR1 } & \text { Fibroblast growth factor receptor 1 } \\ \text { GC } & \text { Gastric cancer } \\ \text { EMT } & \text { Epithelial-mesenchymal transition } \\ \text { TAK1 } & \text { TGF- } \beta \text {-activated kinase 1 } \\ \text { MAPK } & \text { Mitogen-activated protein kinase } \\ \text { AMPK } & \text { AMP-activated protein kinase } \\ \text { mTOR } & \text { Mammalian target of rapamycin } \\ \text { PRKAA1 } & \text { Protein kinase AMP-activated catalytic subu- } \\ & \text { nit alpha 1 } \\ \text { p-ERK } & \text { Phosphorylation of extracellular signal-regu- } \\ & \text { lated kinase } \\ \text { qRT-PCR } & \text { Quantitative real-time polymerase chain } \\ & \text { reaction } \\ \text { FISH } & \text { Fluorescent in situ hybridization }\end{array}$




\section{Introduction}

Systemic chemotherapy and targeted drugs are critical in determining long-term survival in patients with gastric cancer (GC), especially advanced and metastatic cases. However, drug resistance remains a major challenge in GC treatment [1]. The human fibroblast growth factor receptor (FGFR) family, composed of four distinct isoforms (FGFR1-4), belongs to the receptor tyrosine kinase (RTK) superfamily [2,3]. FGFR binds to a fibroblast growth factor (FGF), triggering the dimerization of extracellular receptor domains, trans-phosphorylation of the intracellular kinase domain, and subsequent activation of downstream signaling pathways, mitogen-activated protein kinase (MAPK), and phosphoinositide-3-kinase (PI3K)/AKT [4-6]. FGF/FGFR signaling is involved in numerous physiological processes, including cellular proliferation, migration, and survival [7, 8]. Aberrations in this pathway have been observed in several solid organ cancers [9]. FGFR1 amplification has been observed in oral squamous cell carcinoma, ovarian carcinoma, breast carcinoma, bladder carcinoma, squamous cell lung cancer, pancreatic adenocarcinoma, and gastric adenocarcinoma [10-15], presenting FGFR1 as a promising drug target in cancer therapy [4]. To date, several FGFR inhibitors have been evaluated in clinical trials, with the gradual development of resistance to some inhibitors [8]. Thus, elucidating mechanisms of drug resistance is crucial for further developing FGFR inhibitor therapies.

Here, we observed that the resistance of GC cells to AZD4547, a widely used selective FGFR inhibitor, is associated with an autophagic activity. Autophagy is an evolutionarily conserved catabolic process during which portions of the cytosol and organelles are sequestered into a double-membrane vesicle and delivered to the lysosome for bulk degradation $[1,16]$. The adenosine 5 '-monophosphate (AMP)-activated protein kinase (AMPK)/mammalian target of rapamycin (mTOR) signaling pathway has emerged as a central conduit in autophagic regulation [17]. mTOR can be suppressed by AMPK, stimulating autophagy, and activating the downstream autophagy cascade $[18,19]$. Transforming growth factor- $\beta$ (TGF- $\beta$ )-activated kinase 1 (TAK1), a member of the mitogen-activated protein kinase family [20], plays significant roles in cell differentiation, apoptosis, survival, and inflammatory responses [21]. Growing evidence suggests that TAK1 activation can induce autophagy in an AMPK-dependent manner [22-24]. Here, we observed that both mTOR and TAK1 pathways regulate autophagy in the AZD4547-resistant GC cells and the combined effects regulate autophagy, ultimately increasing epithelial-mesenchymal transition (EMT) effects. Our results indicate that, in FGFR inhibitor-resistant GC patients, TAK1 inhibition could provide a potential therapeutic synergic strategy.

\section{Materials and methods}

\section{Patients and tissue specimens}

Fresh tumors were acquired from surgical resection of patients with histologically-confirmed gastric adenocarcinoma at the Affiliated Cancer Hospital of Nanjing Medical University, China. The histological grade was classified according to the World Health Organization (WHO) histological grading: G1 well-differentiated; G2 moderately differentiated; G3 poorly differentiated. Nerve or vascular invasion was considered neurovascular invasion positive. TNM staging was conducted according to the seventh edition of the AJCC TNM staging system. In total, 34 patients were assigned to the overexpression group according to immunohistochemical results; 16 patients were assigned to the normal expression group. None of the enrolled patients had previous antitumor therapy. The corresponding tumorfree $(2-5 \mathrm{~cm}$ adjacent to the tumor tissue, peripheral; $>5 \mathrm{~cm}$ adjacent to the cancer tissue, normal) tissues were verified by pathological diagnosis.

\section{Animal model and in vivo drug treatments}

Female BALB/c nude mice, aged 5-6 weeks, were purchased from the Medical Laboratory Animal Center of Nanjing Medical University. BGC-823, BGC-823R, MGC-803, or MGC-803R cells $\left(5 \times 10^{6}\right.$ in $0.1 \mathrm{~mL}$ of PBS) were subcutaneously injected into the right flanks of $\mathrm{BCLB} / \mathrm{c}$ nude mice. Tumor volumes were measured using a caliper every 3 days and calculated by the formula: Volume $=0.5 \times$ length $\times$ width $^{2}$. When tumor xenograft volumes reached $\sim 100 \mathrm{~mm}^{3}$ for BGC-823/BGC-823R and $\sim 50 \mathrm{~mm}^{3}$ for MGC-803/MGC-803R, the mice were randomly grouped ( $n=5$, per group) and treated with control vehicle, AZD4547, or AZD4547 combined with NG25. AZD4547 $(12.5 \mathrm{mg} / \mathrm{kg} /$ days $)$ or an equal volume of vehicle (1\%-Tween80 in demineralized water) were administered once daily by oral gavage. NG25 (30 $\mu \mathrm{M} / \mathrm{kg} /$ days) or an equal volume of vehicle $(4 \%$ DMSO + 30\% PEG300 + 5\% Tween- $80+61 \%$ demineralized water) was administered once daily by intraperitoneal injection. After treatment for 15 days, the mice were sacrificed. The tumors were separated, photographed, weighed, and fixed in a formalin solution.

\section{Patient tumor ex vivo organotypic culture}

Tumor tissue samples were collected at the time of surgical removal from consenting patients and transported in Modified Improved Minimum Essential Medium (IMEM, 10\% FBS, and $1 \%$ penicillin-streptomycin (PS). The tissue was sliced into thin sections using a surgical knife. Sections were 
cultured on an organotypic insert (PICM0RG50, Millipore, Darmstadt, Germany) for $24 \mathrm{~h}$ in IMEM, $10 \%$ FBS, $1 \%$ PS, and $50 \mu \mathrm{g} / \mathrm{mL}$ holo-transferrin with the drug. To confirm tissue viability, a section from each tumor was immediately fixed in $10 \%$ buffered formalin. After culture, treated tissue sections were fixed in $10 \%$ buffered formalin and embedded in paraffin. Paraffin-embedded tumors were evaluated for morphology (Hematoxylin-Eosin staining, H\&E) and immunofluorescent signaling.

\section{Statistics}

Comparisons of categorical variables in Supplementary Table S1 were conducted using the Chi-square test. The data are presented as the means \pm standard error of the mean (SEM). Unpaired numerical data were compared using an unpaired $t$ test (two groups) or analysis of variance (ANOVA) for more than two groups. A $p$ value $<0.05$ was regarded as significant.

More materials and methods are provided in Supplementary files.

\section{Results}

\section{FGFR1 is highly expressed in GC}

To investigate the contribution of the FGFR in GC, we compared mRNA levels of each of the FGFR family members in GC tissues and adjacent normal gastric tissues from 50 GC patients by qRT-PCR. FGFR1 was the only significantly upregulated member in GC tissues (Fig. 1a, Supplementary Fig. S1a). Additionally, the protein levels of FGFR1 in GC tissues (T) were consistent with the mRNA levels, which were higher in GC tissues than in non-tumor tissues (N) (Fig. 1b). Our results were further confirmed by IHC analysis in 50 GC samples; the clinical characteristics of GC patients are summarized in Supplementary Table S1. Most (34/50) GC samples exhibited abnormal positive FGFR1 immunostaining, with corresponding normal gastric tissues demonstrating low FGFR1 staining (Fig. 1c).

Thus, we focused on FGFR1 in the pathogenesis and progression of GC. Using the Pearson $\chi 2$ test, we analyzed the correlation of FGFR1 expression with clinical pathologic variables in GC and observed positive correlations between FGFR1 expression levels and histological grade, TNM stages, and lymph node metastasis (Supplementary Table S1; $p<0.05$ and Supplementary Fig. S1b). No significant associations were observed between FGFR 1 and other clinical characteristics, including age and gender. Representative IHC staining images of GC and normal gastric tissues from the Human Protein Atlas (HPA, www.proteinatlas.org) are shown in Supplementary Fig. S1c. Samples presenting antibody staining/protein expression levels of high, medium, low, or not detected were determined by gray-scale colorcoding. This showed undetectable expression levels of FGFR1 in the normal gastric tissues, while GC tissues expressed high levels of FGFR1. To assess whether FGFR1 gene amplification increased in GC tissues, we performed fluorescence in situ hybridization (FISH) analysis (Fig. 1d). The FGFR1 gene was amplified in 5.9\% (2/34) of FGFR1overexpressing GC but not in the FGFR1-negative GC.

Kaplan-Meier analysis of The Cancer Genome Atlas (TCGA) dataset and our central data revealed that high levels of FGFR1 mRNA were associated with poor overall survival (OS) and disease-free survival (DFS) in patients with GC, while no survival correlation was observed for FGFR2, FGFR3, and FGFR4 (Fig. 1e, Supplementary Fig. S1d). Moreover, overexpression of FGFR1 was related to survival probability in TNM stages III-IV, but not in I-II (Supplementary Fig. S1e). Collectively, these data indicated that FGFR1 was highly expressed and associated with poor clinical outcomes in patients with GC.

\section{A subclonal population of FGFR inhibitor-resistant cell models was established}

In GC tissues, FGFR 1 overexpression indicated that FGFR1 might be a therapeutic target in GCs. We first tested the FGFR1 expression in seven GC cell lines by western blotting. The cell lines, SNU-216, MGC-803, BGC-823, and SGC-7901, expressed FGFR1, whereas MKN-45 and HGC27 barely expressed it (Supplementary Fig. S2a). QRT-PCR assays demonstrated similar results, and other FGFR family members were detected at lower mRNA expression levels than FGFR1 in most of GC cell lines (Supplementary Fig. S2b). Next, we assessed the sensitivity of four GC cell lines, SNU-216, MGC-803, BGC-823, and SGC-7901, to AZD4547 using the colony formation assay. Only SNU-216 and SGC-7901 responded well to the inhibitor (Fig. 2a, Supplementary Fig. S2c). Therefore, in BGC-823 and MGC-803 cell lines, we predicted the presence of a subpopulation of cells with primary resistance to FGFR1 inhibition because of the apparent inability of FGFR1 inhibitors to fully reduce cell proliferation and growth. This finding was confirmed by analysis of cell viability using the CCK8 assay. AZD4547 treatment suppressed the proliferative activity of SNU-216 and SGC-7901, but not BGC-823 and MGC-803 cell lines (Supplementary Fig. S2d).

We determined half-maximal inhibitory concentrations (GI50 values) of the AZD4547-resistant cell lines, BGC823 and MGC-803, against AZD4547. GI50 values ranged between 50 and $500 \mathrm{nmol} / \mathrm{L}$ and $100-650 \mathrm{nmol} / \mathrm{L}$ for the BGC-823 and MGC-803 cell lines, respectively. Therefore, we treated BGC-823 and MGC-803 cells with cycles of $1 \mu \mathrm{mol} / \mathrm{L}$ of AZD4547 to isolate resistant cell populations. 

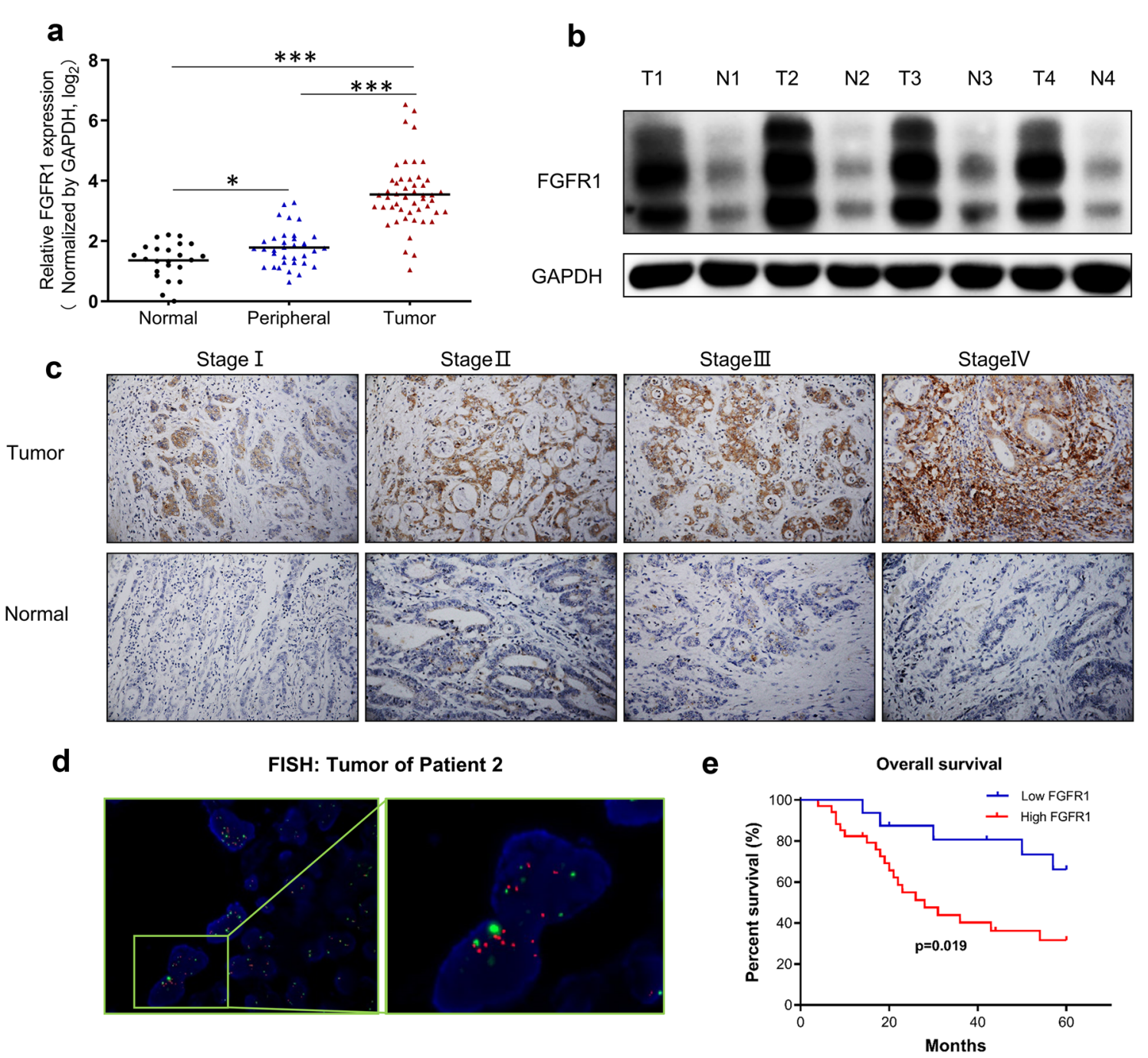

Fig. 1 FGFR1 was highly expressed in gastric cancer (GC) and correlates with poor prognosis in patients with GC. a QRT-PCR of FGFR1 mRNA levels in human normal $(n=23)$, peripheral $(n=35)$, and gastric cancer tissues $(n=50)$. $* p<0.05, * * * p<0.001$. b Western blot analysis was used to detect FGFR1 protein expression in four representative pairs of tumor (T) tissues and adjacent non-tumorous (N) tissues from patients with GC. c Immunohistochemical staining of FGFR1 in stages I-IV of GC tissue samples compared with

After 10-12 weeks of culture, the cells were entirely resistant to AZD4547 (Supplementary Fig. S2e). After $96 \mathrm{~h}$ of treatment with 0.1-30 $\mu \mathrm{mol} / \mathrm{L}$ AZD4547, the resistant BGC823R, and MGC-803R cells exhibited only subtle changes in viability and caused a plateau in the dose-response curves (Fig. 2b).

\section{Drug resistance of cancer cells significantly enhanced survival, migration, and invasion}

To investigate the effects of drug resistance on the biological behavior of GC cells, we compared AZD4547-resistant paired normal mucosae. d FGFR1 amplification was determined by FISH staining of $50 \mathrm{GC}$ tumors. Representative microscopic FISH images at $\times 100$ magnification with high FGFR1 expression according to IHC results. e High expression of FGFR1 predicts poor prognosis of patients with GC in our center. Overall survival (OS) is significantly shorter in patients with FGFR1-high tumors than in those with FGFR1-low tumors $(p=0.019, \log$-rank test $)$

cell lines, BGC-823R and MGC-803R, with their parental cells. Drug resistance significantly increased their malignant behaviors, including anti-apoptosis ability (Fig. 2c), migration, and invasion, with AZD4547 treatment. The number of migrated and invasive BGC-823R and MGC-803R cells was higher than that of the parental groups, especially after inhibitor treatments (Fig. 2d, e). After incubation with the physical-wound and culture in a serum-free medium to exclude the interference of proliferation, parental cells failed to close the wound within $48 \mathrm{~h}$, while resistant cells exhibited greater migration ability (Fig. 2f). Additionally, drug resistance impacted the EMT phenotypes of GC cell lines. 
a

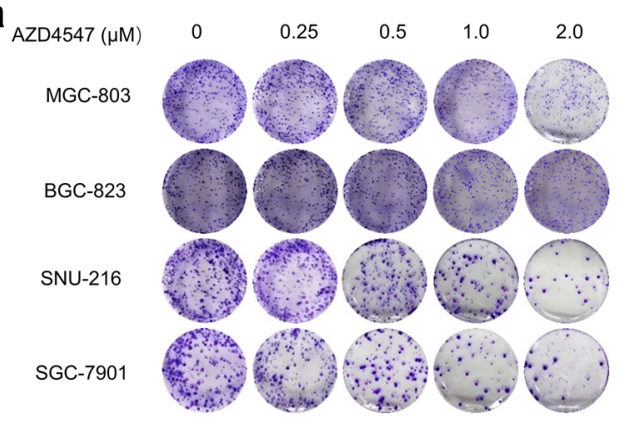

C
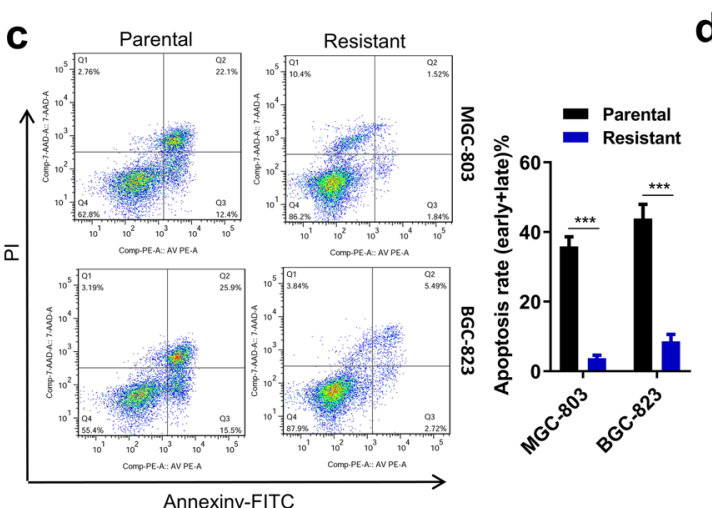

b

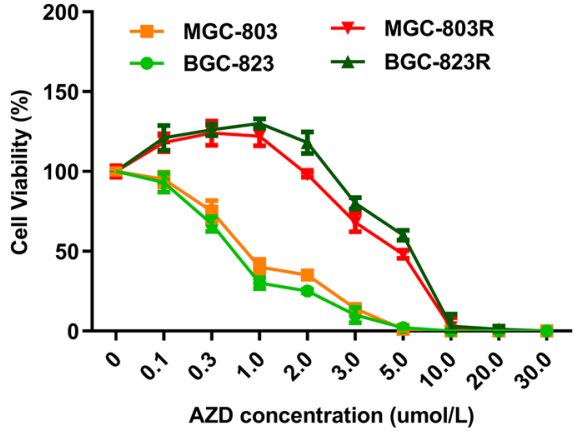

d
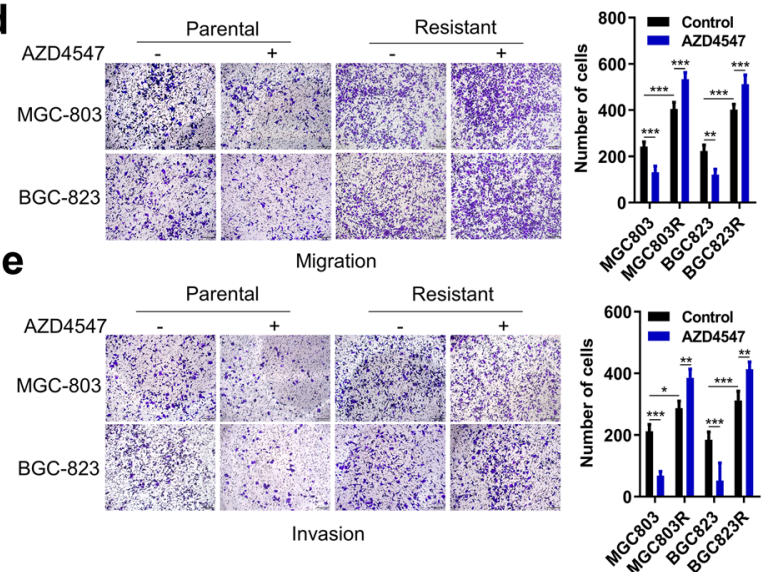
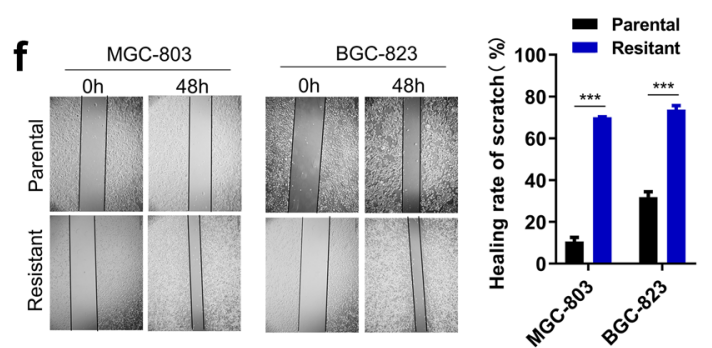

g

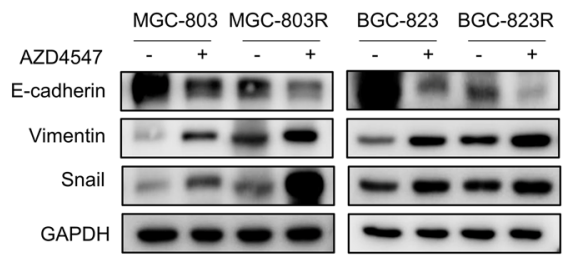

h

MGC-803R
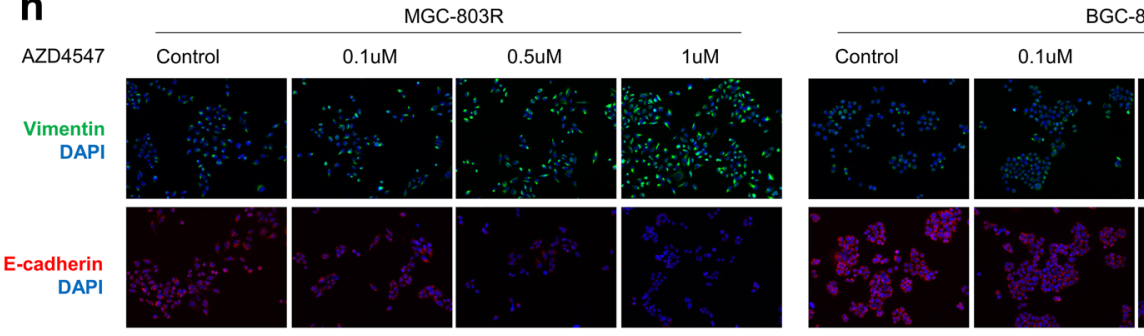

GC-823R
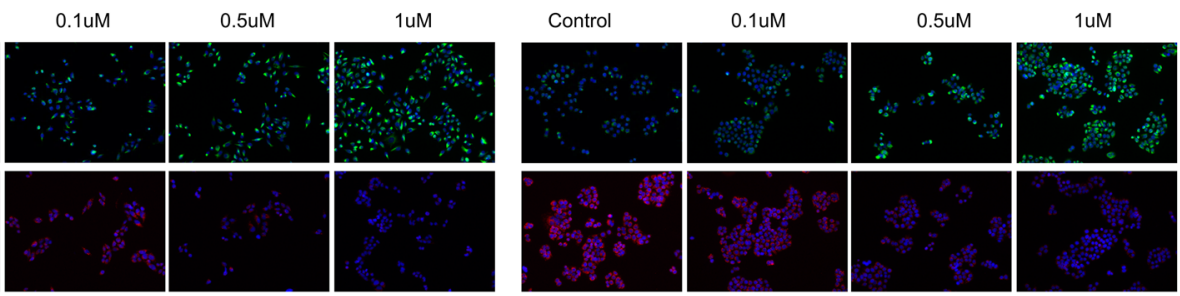

Fig. 2 AZD4547 resistance significantly reduces apoptosis and promotes migration and invasion of GC cells. a Drug sensitivity of FGFR1 expressing cell lines to AZD4547 was determined using the colony formation assay. Cells were treated with increasing concentrations of AZD4547 as indicated for $10-14$ days $(n=3)$. b FGFR dependency was evaluated in the parental cell lines, MGC-803 and BGC-823, and resistant cell lines, MGC-803R and BGC-823R, using AZD4547 by CCK8 measurement after $96 \mathrm{~h}$. c Apoptosis detected by Annexin V-PI assays. d, e Representative images and quantification results of cell migration (d) and invasion (e) abilities of control or

Western blot analysis revealed reduced E-cadherin levels, while vimentin and Snail were augmented in the BGC-823R and MGC-803R cells when compared with the parental resistant cells. f Representative images and analysis of wound repair at $0-48 \mathrm{~h}$ after scratch wounding. $\mathbf{g}$ Western blotting analysis of epithelial and mesenchymal protein expression after AZD4547 treatment for $48 \mathrm{~h}$. h Immunofluorescence analysis via laser confocal microscopy indicates that the EMT process is induced by different concentrations of AZD4547 treatment. Each experiment was performed in triplicate. Data are presented as the means \pm SEM and analyzed with Student's $t$ test $\left({ }^{*} p<0.05,{ }^{* *} p<0.01,{ }^{* * *} p<0.001\right)$. EMT, epithelialmesenchymal transition

cells, especially after AZD4547 treatment (Fig. 2g). Confocal immunofluorescence analysis confirmed a dose-dependent transition after $48 \mathrm{~h}$ of AZD4547 treatment (Fig. 2h). 
These results indicated that drug resistance was associated with the malignant phenotypes.

\section{Resistance of GC cells to AZD4547 was associated with autophagic activity}

To understand the mechanism of primary AZD4547 resistance in GC cells, we performed a whole-transcriptome shotgun sequencing to determine the continuous cellular transcriptome changes between parental and resistant cells, treated with $1 \mu \mathrm{mol} / \mathrm{L}$ AZD4547 in the presence of $25 \mathrm{ng} /$ mL FGF2 with two cell lines, MGC-803 and BGC-823. Furthermore, we conducted the RNA-Seq for each cell line in triplicate. Supervised hierarchical clustering revealed that the expression levels of 586 genes significantly differed between parental and resistant MGC-803 cells: 285 genes were upregulated and 301 genes were downregulated, while there were 230 up-regulated and 271 down-regulated genes in BGC-823 cells group (Fig. 3a, b and Supplementary Fig. S3a, b).
All differently expressed gene transcripts from MGC803 cells group were further analyzed using the Database for Annotation, Visualization and Integrated Discovery (DAVID) Bioinformatics Resources (https://david.ncifc rf.gov), with the results demonstrating that the most pronounced differences were associated with morphologic abnormalities, altered substrate metabolism, and autophagy (Fig. 3c). The PI3K-Akt-mTOR pathway is known to be the main regulator of autophagy. Similar results were also observed that differentially expressed genes (DEGs) were mainly enriched in the positive regulation of autophagy and PI3K/AKT signaling pathway by DAVID analysis of the RNA-seq data from BGC-823 cells group (Supplementary Fig. S3c). The Gene Set Enrichment Analysis (GSEA) showed that the gene sets of the ENERGY_DEPENDENT_ REGULATION_MTOR_BY_LKB1_AMPK, EMT, and POSITIVE_REGULATION_OF_AUTOPHAGY pathways were significantly enriched in AZD4547-resistant MGC803R cells when compared with parental cells, indicating that these gene sets could be significantly associated with

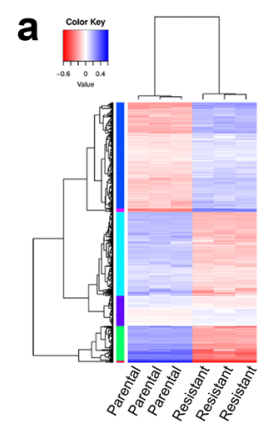

b

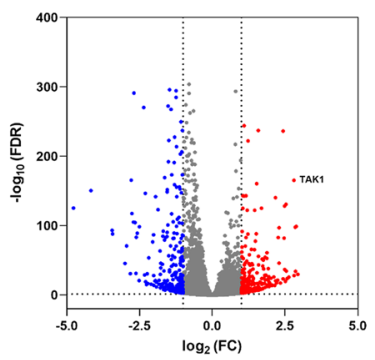

c

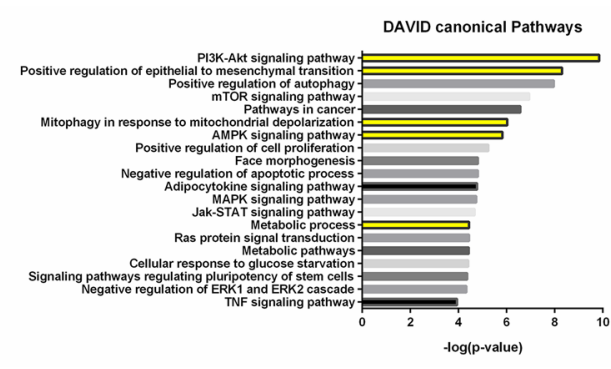

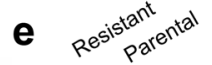

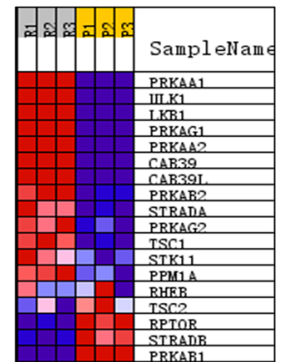

d
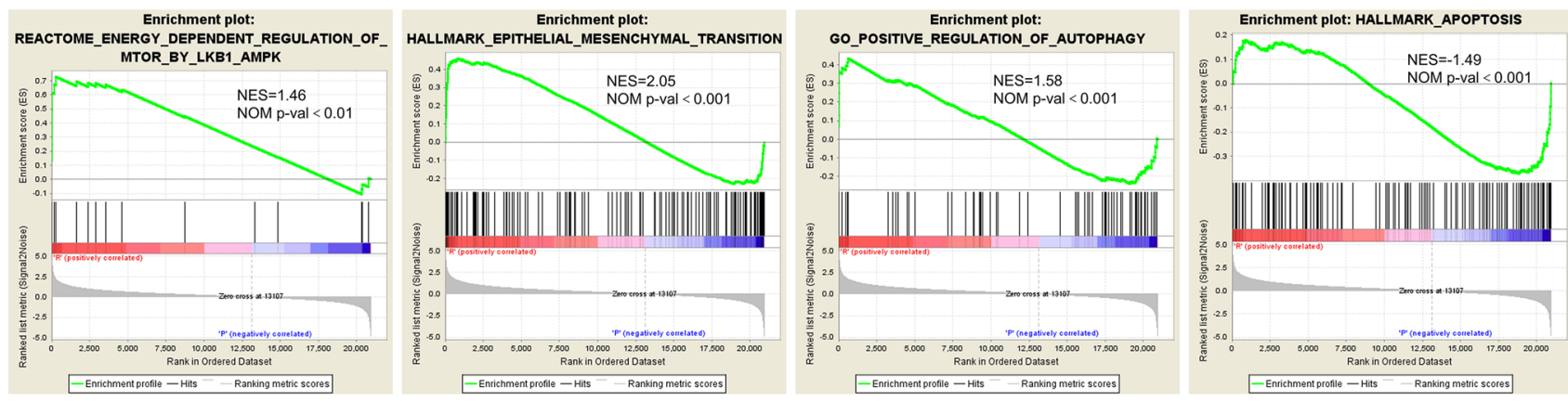

Fig. 3 Landscape of mRNA expression in parental and AZD4547resistant MGC-803 cells. a Hierarchical clustering heat maps of the significantly differentially expressed genes from RNA-Seq in parental MGC-803 and AZD4547-resistant MGC-803R cells with three technical replicates. The expression values are shown in shades of blue and red, indicating high expression and low expression across all the samples respectively. b Volcano plot showing differentially expressed genes between the MGC-803 and MGC-803R cells. Each red or blue dot denotes an individual gene with $F D R \leq 0.05$ (two-sided moderated $t$ test with limma) and fold change $>2$. Red: upregulation, Blue: downregulation. c KEGG pathways and gene ontology (GO) biologi-

cal process observed from DAVID analysis of relatively active genes due to AZD4547 treatment resistance. d GSEA analysis of the significant pathways of differentially expressed protein-coding genes set in two groups. The top portion of the figure plots the enrichment scores for each gene, whereas the bottom portion of the plot shows the value of the ranking metric moving down the list of ranked genes. $Y$-axis: value of the ranking metric; $X$-axis: rank for all genes; NES: normalized enrichment score. e mRNA levels of the related genes in the mTOR signaling pathways in MGC-803 versus MGC-803R cells. Red: high expression; Blue: low expression 
resistant expression in GC, while the APOPTOSIS pathway was enriched in parental cells (Fig. 3d). Furthermore, we explored related genes in the mTOR signaling pathways, with the AMPK/MTOR axis demonstrating the most significant differential expression in the pathways (Fig. 3e). As the gene expression profile revealed major differences related to mitochondrial dysfunction and altered morphology, we hypothesized that the regulation of autophagy would be affected by drug resistance. To determine whether FGFR1 inhibitors could induce autophagy to sustain drug-tolerant cells, we evaluated the conversion of autophagy markers, LC3B, and SQSTM1/p62, using western blotting. The intracellular levels of LC3B were increased, and the p62 levels were decreased in MGC-803R and BGC-823R cells, indicating an enhanced autophagic process (Fig. 4a). To further investigate the flux through the autophagic pathway, we constructed a lentivirus containing monomeric red fluorescent protein (mRFP)-green fluorescent protein (GFP)-LC3 monitoring autophagy [25]. Cells with increased autophagic influx were expected to demonstrate increased yellow signals because of the fusion of GFP with the lysosomes. As shown in Fig. 4b, when drug-resistant cells were treated with AZD4547, increased yellow signals were detected when compared with the control cells. Collectively, these results indicated that resistant cells exhibited a higher level of autophagy, and AZD4547 induced enhanced autophagic flux than the parental human GC cell lines.

\section{The induction of autophagy in AZD4547-resistant GC cells was mediated through activating the AMPKa1/mTOR pathway}

Based on pathway enrichment analysis via the Kyoto Encyclopedia of Genes and Genomes (KEGG) and GSEA, we hypothesized that AZD4547 exerted its effects on resistant GC cells through AMPK/mTOR signaling (Fig. 3c, d). Following $1 \mu \mathrm{mol} / \mathrm{L}$ of AZD4547 treatment, we confirmed that all resistant cells exhibited significant AMPK phosphorylation. Furthermore, resistant cells showed inhibited mTORC1 (as seen by a decrease in phosphorylated S6), whereas total S6 retained a consistent expression level (Fig. 4a). Mutations in the FGFR1 open reading frame that might induce resistance were not detected from RNA-Seq results (data not shown). All AZD4547-resistant cells displayed sustained AMPK activation with drug therapy, thus indicating possible AMPK/mTOR pathway activation and autophagy induction in mediating the tumor EMT process and metastases. We observed that AZD4547 was able to induce autophagy in a dose-dependent manner in BGC-823R and MGC-803R cells, as seen by the lipidation of LC3 and low activation of phosphorylated S6 (Fig. 4c).

Protein kinase AMP-activated catalytic subunit alpha 1 (PRKAA1) is a catalytic subunit of AMPK, also known as
AMPK $\alpha 1$, required for the formation of the AMPK heterotrimeric serine/threonine kinase complex with the $\beta$ - and $\gamma$-subunits. PRKAA1 plays a key role in regulating cellular energy metabolism and modulates autophagy programs [26]. We assessed the expression of LC3B, S6, and phosphorylation of S6 (p-S6) following AZD4547 treatment in BGC823R and MGC-803R cells, and observed that AZD4547 minimally induced autophagy when PRKAA1 was knocked down by siRNA (Fig. 4c, Supplementary Fig. S4a, b). In contrast, LC3-II in parental BGC-823 and MGC-803 cells was not increased following AZD4547 treatment after transfection with PRKAA1 siRNA interference, further confirmed in PRKAA1-deficient BGC-823 cells (PRKAA1 ${ }^{-/}$) via the CRISPR-Cas9 System (Fig. 4d, Supplementary Fig. S4c, d). Likewise, AZD4547-induced autophagy was abrogated by the AMPK inhibitor, Compound C, in BGC-823R and MGC-803R cells, while simultaneously rescuing phosphorylated S6 levels (Fig. 4e).

Confocal microscopy after mRFP-GFP-LC3 transfection demonstrated enhanced autophagic flux following the treatment of AZD4547-resistant GC cells with AZD4547. However, yellow signals were attenuated after Compound C treatment alone or in combination with AZD4547 (Fig. 4f). These results indicated that the FGFR1 inhibitor, AZD4547, increased AMPK activation to reduce mTOR signaling, resulting in autophagy in AZD4547-resistant cells, but not in parental cells.

\section{Metabolic alteration activated the AMPK/mTOR pathway by AZD4547 treatment-induced ERK phosphorylation}

AZD4547-resistant GC cells, BGC-823R and MGC-803R, exhibited effective activation of AMPK/mTOR signaling; hence, we speculated whether metabolic alterations sustain the survival of cancer treated cells, as glucose uptake and metabolism affect multiple steps in AMPK/mTOR pathway. Reportedly, AZD4547 effectively inhibits the phosphorylation of FGFR1 and mitogen-activated protein kinase (MEK)/ extracellular signal-regulated kinase (ERK) signaling in FGFR1-amplified cancers [27, 28]. As expected, a $24 \mathrm{~h}$ treatment with AZD4547 suppressed ERK phosphorylation (p-ERK) in resistant cells, resulting in the activation of AMPK. Notably, in parental MGC-803 and BGC-823 cell lines, total ERK was observed at a consistent expression level, with sustained suppression of p-ERK in the presence of the drug (Fig. 5a). Therefore, we hypothesized that AZD4547 treatment would negatively impact glucose uptake and, subsequently, the biosynthetic pathways, resulting in a drug-enforced reduction in glucose availability in resistant cells.

To determine the effects of AZD4547 treatment on the regulation of metabolism, we measured glucose 


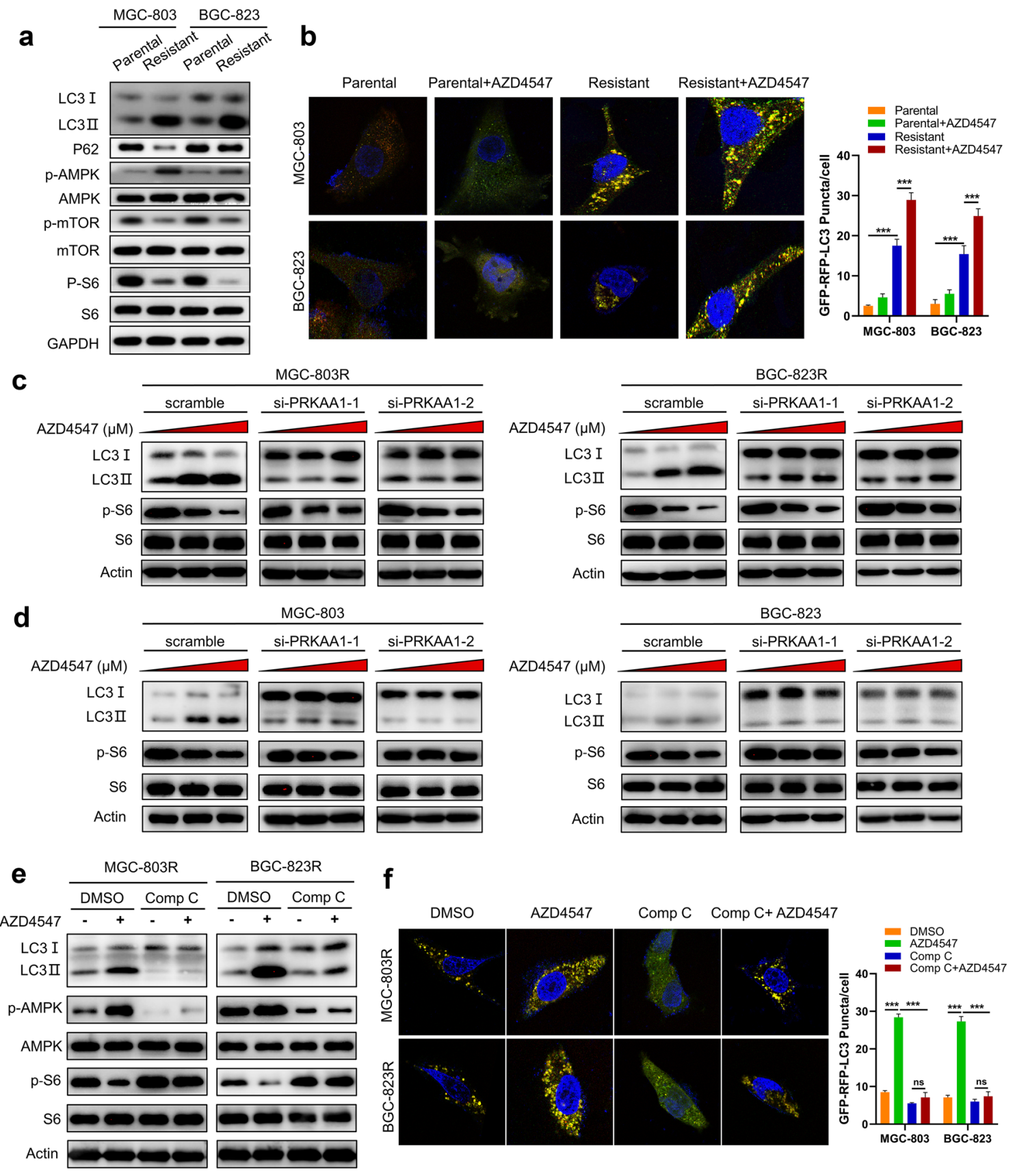

Fig. 4 AZD4547-resistant cells exhibit an autophagic process via the AMPK-mTOR pathway. a MGC-803 and BGC-823 cells, as well as resistant cells, were treated with $1 \mu \mathrm{M}$ AZD4547 for $24 \mathrm{~h}$ and immunoblotted with LC3, p62, p-AMPK, total AMPK, p-mTOR, total mTOR, p-S6, total S6, and GAPDH. b The GFP and RFP signals of tandem fluorescent LC3 (RFP-GFP-LC3) show different localization patterns. Cells were transfected with plasmids expressing either tandem fluorescent LC3. $24 \mathrm{~h}$ after transfection, the cells were treated with $1 \mu \mathrm{M}$ AZD4547, fixed, and analyzed by microscopy. Representative cells were visualized under a confocal microscope (left) and statistically analyzed (right). Bar diagram (mean \pm SEM) representing the average "yellow only" spot counts/cell. c MGC-803R and BGC-823R were treated with 0-3 $\mu \mathrm{M}$ AZD4547 for $24 \mathrm{~h}$ after transiently transfected with siRNA against PRKAA1. LC3 processing and mTOR activity were evaluated by immunoblotting. d MGC-
803 and BGC-823 were treated with 0-3 $\mu \mathrm{M}$ AZD4547 for $24 \mathrm{~h}$ after transiently transfected with siRNA against PRKAA1. LC3 processing and mTOR activity were evaluated by immunoblotting. e MGC-803R and BGC-823R cell lysates in the absence or presence of AZD4547 $(1 \mu \mathrm{M})$ and Compound $\mathrm{C}(1 \mu \mathrm{M})$ were prepared and analyzed to determine LC3 levels and AMPK ${ }^{\mathrm{T} 172}$ and S6 phosphorylation by western blot with the indicated antibodies or the antibodies against total proteins after stripping. $\beta$-actin was used as a loading control. f Compound C inhibits AZD4547 resistant-induced formation of autolysosomes. The cells stably expressing the GFP-RFP-LC3 gene were treated with AZD4547 or DMSO for $24 \mathrm{~h}$ in the absence or presence of CC. The cells were then fixed and stained with DAPI. Each experiment was performed in triplicate. The data are presented as the means \pm SEM and analyzed with Student's $t$ test $\left({ }^{*} p<0.05\right.$, $* * p<0.01, * * * p<0.001)$ 

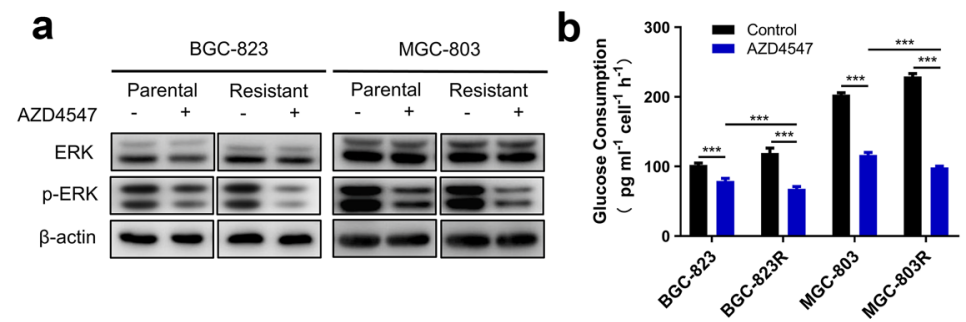

d

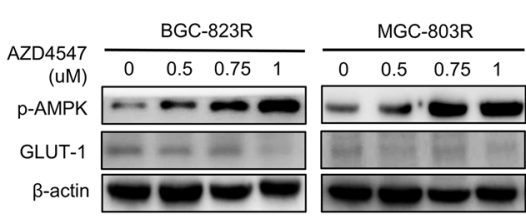

e

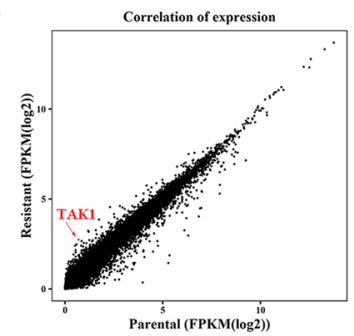

C

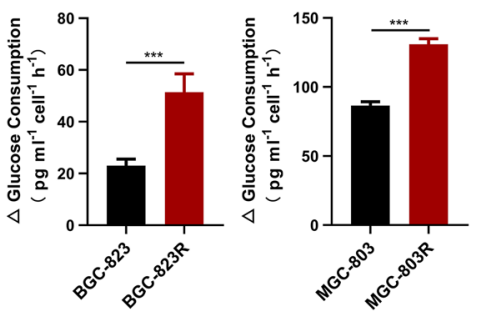

f

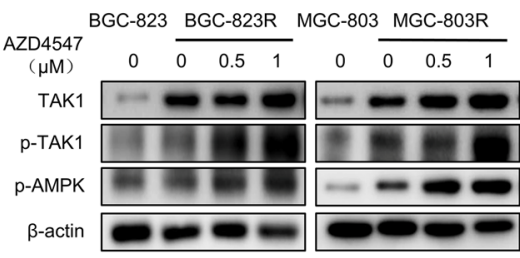

g

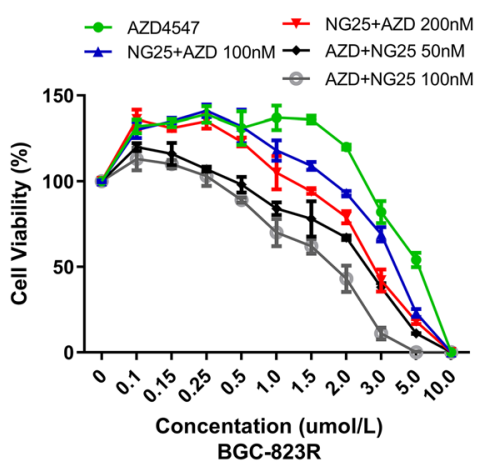

h

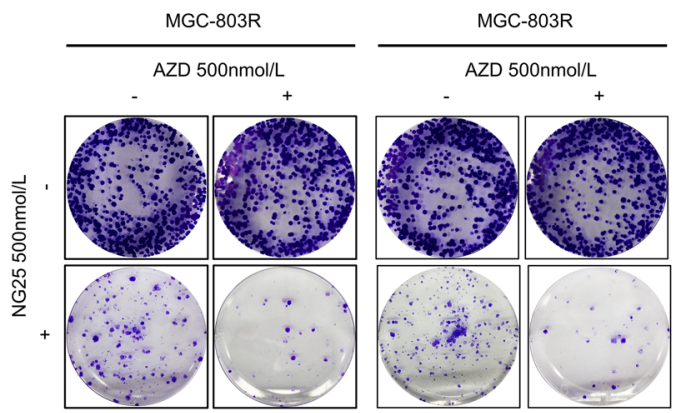

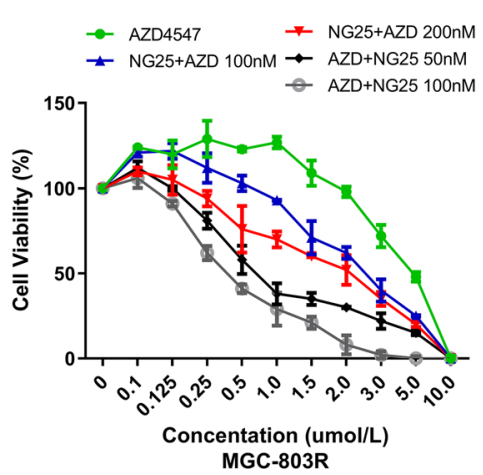

i

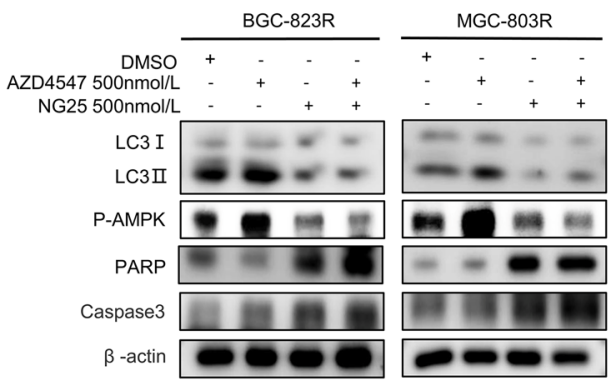

Fig. 5 The effects of AZD4547 treatment on metabolism and TAK1 overexpression as a resistance factor in BGC-823 and MGC-803 cells. a BGC-823 and BGC-823R and MGC-803 and MGC-803R cells were untreated or treated with $1 \mu \mathrm{M}$ AZD4547 for $24 \mathrm{~h}$. Protein levels of p-ERK, ERK, and $\beta$-actin were measured by western blot assays. b Glucose levels in the culture medium were measured in control and AZD4547-treated cells. Glucose consumption was calculated and normalized to cell number. c Decreased glucose consumption for parental and resistant cells after AZD4547 treatment $(\Delta$ glucose consumption $=$ glucose consumption of control - glucose consumption after AZD4547 treatment). d BGC-823R and MGC803R cells were treated with the control to $1 \mu \mathrm{M}$ AZD4547 for $24 \mathrm{~h}$ and probed with the indicated antibodies. e MGC-803 and MGC803R expression values generated from RNA-Seq data are plotted as FPKM values. TAK1 FPKM values are highlighted (red). f Lysates of cells treated with the increasing concentrations of AZD4547 were immunoblotted with TAK1, p-TAK1, and p-AMPK. $\beta$-actin was used as a loading control. $\mathrm{g}$ FGFR dependency evaluation in BGC-823R and MGC-803R cells using AZD4547 alone or combined with the TAK1 inhibitor, NG25, for $96 \mathrm{~h}$. The combination was screened using increasing concentrations of AZD4547 (black, gray) or NG25 (blue, red), at constant concentrations of NG25 (50 nM black, $100 \mathrm{nM}$ gray) or AZD4547 (100 nM blue, $200 \mathrm{nM}$ red). h Crystal violet clonogenic assay of BGC-823R and MGC-803R cells. Cells were plated on 6-well plates and treated for $96 \mathrm{~h}$ with DMSO, $500 \mathrm{nM}$ of AZD4547, $\mathrm{NG} 25$, or in combination. i Cells were treated for $24 \mathrm{~h}$ with DMSO, $500 \mathrm{nM}$ of AZD4547, NG25, or in combination with BGC-823R and MGC-803R cells, and protein expression was measured by Western blotting. Data are shown as means \pm SEM of experimental triplicates $(* p<0.05, * * p<0.01, * * * p<0.001 ;$ ns: no significance) 
consumption of parental and resistant cells after AZD4547 treatment. AZD4547 treatment decreased the glucose consumption in AZD4547-resistant cells, as well as the parental cell lines; the decreased glucose consumption was significantly higher in AZD4547-resistant cells than in parental cells, which might be a consequence of the low p-ERK levels in resistant cells (Fig. 5b, c). In resistant GC cells, this impaired carbon metabolism resulted in reduced cell size (Fig. 4b). Importantly, under glucose limitation, we observed increased AMPK phosphorylation at Thr172, an established indicator of metabolic stress, and decreased glucose transporter-1 (GLUT-1), a crucial player involved in glucose uptake and metabolism (Fig. 5d) [29]. These findings indicated the role of AMPK in mitigating metabolic stress and promoting cancer cell survival.

\section{AMPK activation induced AZD4547 resistance partly through overexpression of TAK1}

The above data suggested that decreased glucose uptake, particularly in AZD4547-resistant strains, may be required for AZD4547 responses, and we investigated why highlevel AMPK phosphorylation is associated with distinct correlations with mTOR signaling and autophagy occurrence in drug-sensitive and resistant strains. We analyzed the RNA-seq data of the resistant and parental cells from MGC-803 and BGC-823 cells group. Of the candidate genes, we focused on TAK1 from a cluster of differentially upregulated genes (Fig. 3b, Supplementary Fig. S3b). Transcriptome sequencing revealed at least a 22 -fold transcriptional TAK1 upregulation (Fig. 5e, Supplementary Fig. S5a, b). Emerging evidence suggests that TAK1 activation led to increased phosphorylation of its downstream substrate AMPK $^{\mathrm{T} 172}$ [30]. AMPK phosphorylates multiple serine residues in Unc-51-like autophagy activating kinase 1 (ULK1), a marker of the autophagic initiation complex, activating ULK1 [31, 32]. Interestingly, we observed a strong increase in TAK1, as well its phosphorylation, in resistant cells, suggesting that up-regulation and activation of TAK1 might be associated with resistance to FGFR1 inhibition, similar to other settings of drug resistance (Fig. 5f) [33]. Hence, we cotreated resistant cells with AZD4547 and the TAK1 inhibitor, NG25. The combined treatments led to a depletion of phosphorylated AMPK (p-AMPK) and effective cytotoxicity, while simultaneously increasing the apoptosis phenotype (Fig. 5g-i, Supplementary Fig. S5c). Notably, combined FGFR and TAK1 inhibition demonstrated a highly suppressive effect in AZD4547-resistant cells, supporting a role for TAK1 in resistance to FGFR1 inhibition. Next, we performed in vivo experiments using a subcutaneous xenograft tumor model. The results demonstrated that combined treatments with AZD4547 and NG25 achieved remarkable therapeutic effects in the resistant mice group (Fig. 6a-c,
Supplementary Fig. S6a-c). Further results demonstrated that the combination treatment with AZD4547 and NG25 significantly increased apoptosis (Fig. 6d, Supplementary Fig. S6d).

To evaluate clinical samples, we exposed patient-derived GC organotypic cultures to AZD4547 treatment for $24 \mathrm{~h}$. The patients with GC were subdivided into high and low expression subgroups according to the gene transcription medians of TAK1 in tumors. AZD4547 significantly induced LC3B expression while simultaneously increasing p-AMPK levels in high expression TAK1 patients. Simultaneously, combination treatment with AZD4547 and NG25 resulted in a significant increase in apoptosis (established by an increase in the immunoactivity of TUNEL; $p<0.001$ ) (Fig. 7a, b; Supplementary Fig. S7a, b). These findings indicated TAK1 is activated in FGFR 1 inhibitor-resistant GC cells. In patients with GC, TAK1 expression can serve as a valuable indicator for predicting FGFR1 inhibitor resistance. Additionally, TAK1 expression in GC patients was highly and positively correlated with beclin-1 expression, moderately correlated with LC3B in the TCGA database, which is well-established markers in autophagy (Fig. 7c). Overall, our results demonstrated that TAK1 positively regulates AZD4547 resistance and promotes further invasion and metastasis in GC cells through the AMPK-induced autophagic pathway (Fig. 7d).

\section{Discussion}

Inhibitors targeting FGFR1 provide an attractive prospect for the advancement of GC treatment [34]. However, several patients cannot achieve satisfactory therapeutic effects due to drug resistance [1]. To explore the AZD4547-resistance comprehensively, we established two AZD4547-resistant cell models with GC cell lines MGC-803 and BGC-823, termed MGC-803R and BGC-823R. Notably, FGFR resistance could promote EMT and AZD4547 treatment could induce the EMT process in a dose-dependent manner. These findings are consistent with previous reports, where EMT is related to the resistance of multiple anti-cancer drugs, including chemotherapy $[35,36]$ and targeted therapies $[37$, 38].

To clarify the mechanism of primary AZD4547 resistance in GC cells, RNA-Seq was performed to recognize the cellular transcriptome changes between parental and resistant cells. Based on RNA-Seq analyses, we focused on autophagy and the TAK1-AMPK-mTOR axis, demonstrating that MGC-803R and BGC-823R cells enhanced autophagic activity. Moreover, AZD4547 treatment enhanced autophagic flux in resistant GC cells than their parental cells. Autophagy inhibitors have been shown to sensitize cancer cells to chemotherapy, radiation, tamoxifen 


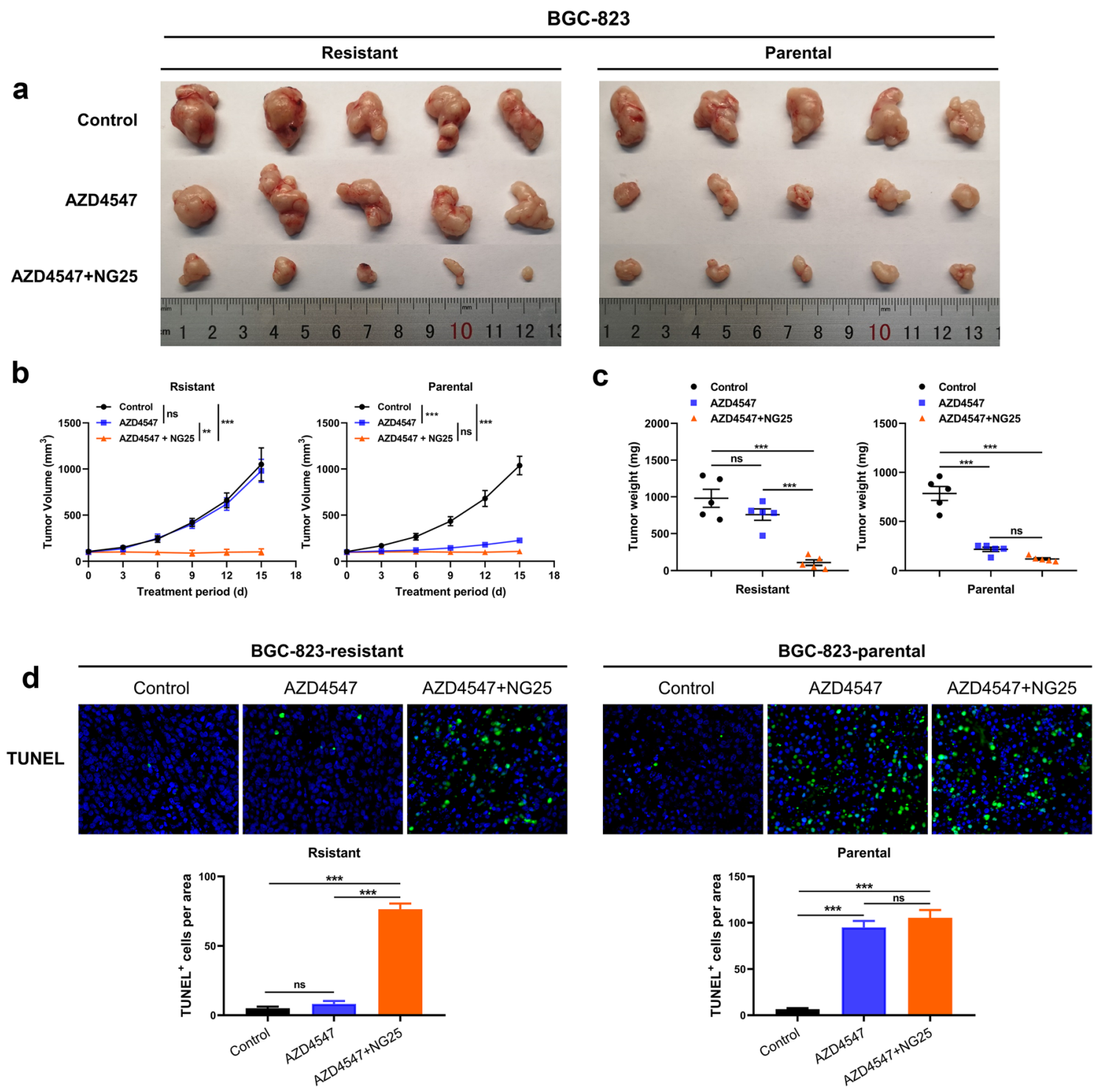

Fig. 6 Combined treatments with AZD4547 and NG25 acquire a significant antitumor effect in vivo. a BGC-823 and BGC-823R cells were inoculated into nude mice to establish subcutaneous xenograft tumors. The mice were sacrificed after treatment for 15 days. The dissected tumors were photographed. Representative macroscopic

treatment, and molecular targeted therapy [39-42], exhibiting the cytoprotective role of autophagy in established tumors. The AMPK/mTOR pathway functions as a central conduit for autophagic signaling pathways to promote cell survival or death [1]. The mTOR pathway negatively regulates autophagy and AMPK can suppress mTORC1 signaling to stimulate autophagy [43]. In our study, MGC-803R and BGC-823R cells exhibited sustained AMPK/mTOR activation and enhanced autophagy activity, which may be crucial for modulating resistance to FGFR1 inhibition. Additionally, our results showed that TAK1 was upregulated in resistant BGC-823R and MGC-803R cells when compared views showed tumors in indicated groups. b Tumor volumes were monitored every 3 days after indicated treatments. $\mathbf{c}$ Dissected tumor weights were measured. d Representative images and quantification of TUNEL staining for tumor tissues from mice. Data are presented as means $\pm \operatorname{SEM}(* p<0.05, * * p<0.01)$

with their parental cells. TAK1 is a serine/threonine kinase crucial for immunoregulation and intracellular signaling pathways [44]. Previous reports have indicated that TAK1 activation could induce autophagy through AMPK activation [30]. TAK1 acts as an AMPK upstream kinase to affect both autophagy and PPAR $\alpha$ activity $[24,45]$. Therefore, we hypothesized that autophagy plays a role in the AZD4547 resistance of GC cells through the activation of TAK1AMPK-mTOR signaling.

Activation of AMPK in BGC-823R and MGC-803R cells, especially after AZD4547 treatment, led us to consider whether metabolic alteration sustains the survival of 


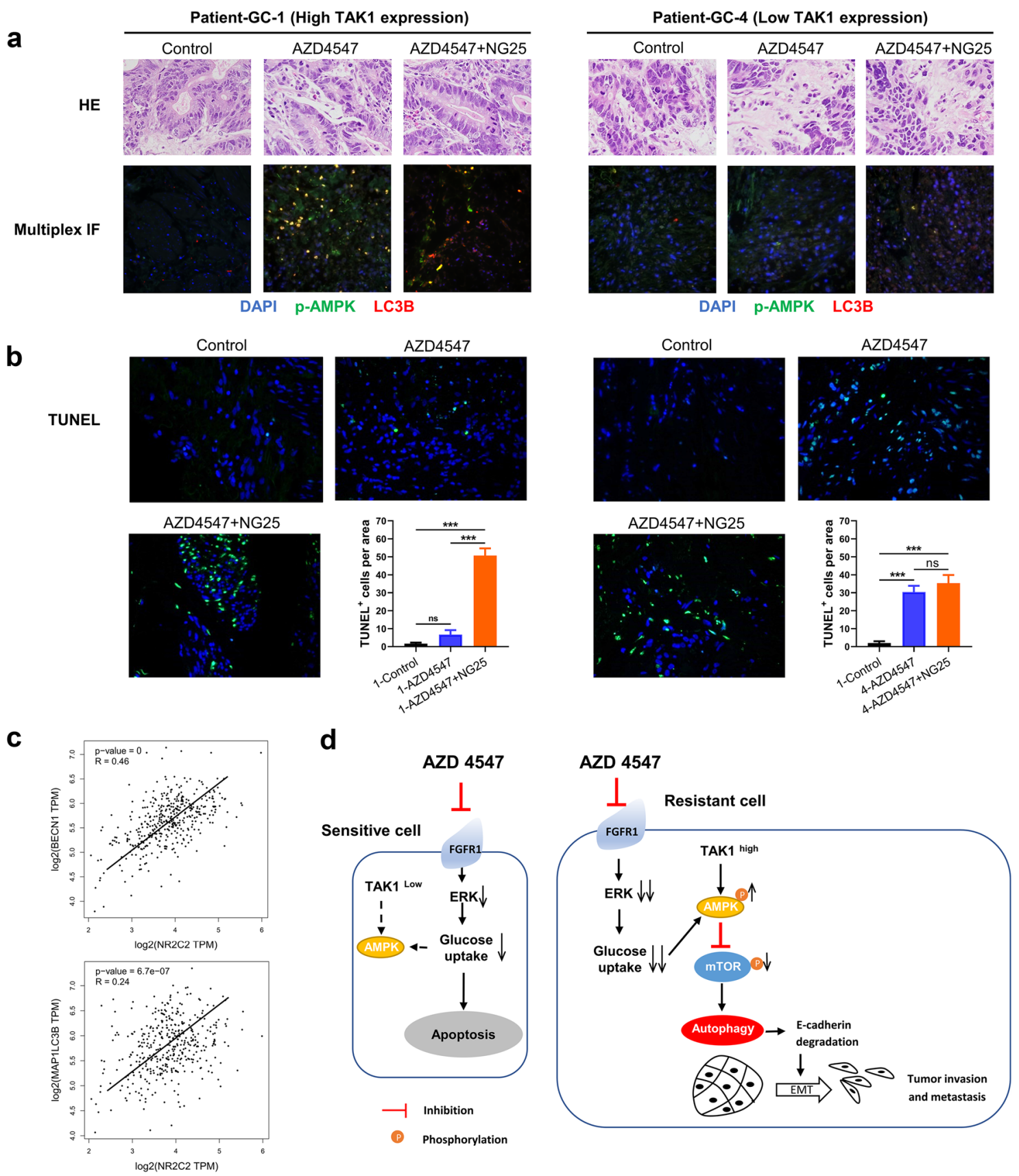

Fig. 7 Anti-cancer effects of AZD4547 and NG25 by the suppression of autophagy-mediated drug resistance in cancer. a Patientderived organotypic cultures $(n=8)$ were treated with AZD4547 for $24 \mathrm{~h}$ and stained with p-AMPK and LC3B (representative images from two different patient tumors with relatively high and low TAK1 expression, respectively). b Representative images and quantification of TUNEL staining in patient-derived organotypic cultures. Each

the resistant cells. Previous studies have reported that the FGFR inhibitor effectively inhibited MEK/ERK signaling in FGFR1-amplified cancers [30, 31]. Additionally, the ERKMAPK signaling pathway has been shown to contribute experiment was performed in triplicate. The data are presented as the means \pm SEM $(* p<0.05, * * p<0.01)$. c Correlations among levels of beclin-1, LC3B, and TAK1 expression in GC tumor were denoted with Pearson's correlation coefficients using the TCGA database. d Schematic diagram of mechanisms: Resistance to FGFR1 targeting therapy leads to autophagy and EMT by metabolic restrictions and TAK1/AMPK activation

to a high rate of glucose uptake [46, 47]. In our study, we demonstrated that AZD4547 suppressed the phosphorylation of ERK and glucose uptake, notably in resistant cells and marginally in parental cells. As a nutrient and energy sensor, 
AMPK maintains energy homeostasis [48]. In AZD4547resistant cells, the inhibition of FGFR1 decreased glucose uptake sharply through ERK, inducing increased AMPK phosphorylation to rapidly regulate metabolic energy balance, further activating the AMPK/mTOR pathway. Double activation of AMPK/mTOR signaling induced by decreased glucose uptake and highly expressed TAK1, which exists only in AZD4547-resistant cells, synergistically enhanced autophagic activity, subsequently promoting EMT and metastasis in resistant GC cells. In parental cells, slightly decreased glucose uptake and low expression of TAK1 were insufficient to activate the AMPK/mTOR pathway adequately, eventually inducing cellular apoptosis.

Combination treatment with AZD4547, an FGFR inhibitor, and NG25, TAK1 inhibitor, in vitro, and in vivo, revealed a remarkable therapeutic effect on the AZD4547resistant cells, with obviously decreased p-AMPK and an increased apoptosis phenotype. Patient-derived GC organotypic cultures and animal models with AZD4547 or AZD4547 + NG25 treatment revealed similar results. Therefore, our findings provide a novel treatment strategy for patients with FGFR inhibitor resistance. Detection of TAK1 levels could help predict whether a patient would develop resistance to FGFR inhibitor and whether combination treatment with the FGFR and TAK1 inhibitors would be necessary.

In summary, the drug-resistant subpopulation of GC cells overexpress and activate TAK1, inducing autophagic activity by activation of the AMPK/mTOR pathway, which eventually results in FGFR1 inhibitor resistance. Furthermore, the resistant subpopulation of GC cells can be effectively treated with combination FGFR1 and TAK1 inhibition.

Acknowledgements This work was supported by Grants from the National Natural Science Foundation $(81772602,91742105$ to YC and 81760553,81960528 to SJZ, 81772569, 81572370 to RQJ), the Jiangsu Provincial key research development program of China (BE2018750 to YC), Key Laboratory of Emergency and Trauma (Hainan Medical University), Ministry of Education (KLET-201913 to YC), the Finance Science and Technology Projects of Hainan Province (2017CXTD008 to SJZ), the Fundamental Research Funds for the Central Universities (14380447 to RQJ) and Grant of Jiangsu Cancer Hospital (ZN201613 to RP).

Author contributions YC, SJZ, and RQJ conceived and designed the experiments. RP, YC, LNW and SRL participated in the experiments and drafted the manuscript. GL contributed to the sample collection and interpretation of the data. LNW and DJF revised the manuscript. All authors read and approved the final manuscript.

\section{Compliance with ethical standards}

Conflict of interest The authors declare that they have no conflict of interest.

Ethical approval All procedures performed in studies involving human participants were in accordance with the ethical standards of the Eth- ics Committee of Nanjing Medical University and with the Helsinki Declaration of 1964 and later versions. All relevant guidelines and regulations for the care and use of laboratory animals were followed.

Informed consent Informed consent to be included in the study was obtained from all patients.

\section{References}

1. Sui X, Chen R, Wang Z, Huang Z, Kong N, Zhang M, et al. Autophagy and chemotherapy resistance: a promising therapeutic target for cancer treatment. Cell Death Dis. 2013;4:e838.

2. Schafer MH, Lingohr P, Strasser A, Lehnen NC, Braun M, Perner $\mathrm{S}$, et al. Fibroblast growth factor receptor 1 gene amplification in gastric adenocarcinoma. Hum Pathol. 2015;46(10):1488-95.

3. Turner N, Grose R. Fibroblast growth factor signalling: from development to cancer. Nat Rev Cancer. 2010;10(2):116-29.

4. Ying S, Du X, Fu W, Yun D, Chen L, Cai Y, et al. Synthesis, biological evaluation, QSAR and molecular dynamics simulation studies of potential fibroblast growth factor receptor 1 inhibitors for the treatment of gastric cancer. Eur J Med Chem. 2017;127:885-99.

5. Carter EP, Fearon AE, Grose RP. Careless talk costs lives: fibroblast growth factor receptor signalling and the consequences of pathway malfunction. Trends Cell Biol. 2015;25(4):221-33.

6. Liang G, Liu Z, Wu J, Cai Y, Li X. Anticancer molecules targeting fibroblast growth factor receptors. Trends Pharmacol Sci. 2012;33(10):531-41.

7. Touat M, Ileana E, Postel-Vinay S, Andre F, Soria JC. Targeting FGFR signaling in cancer. Clin Cancer Res. 2015;21(12):2684-94.

8. Xue WJ, Li MT, Chen L, Sun LP, Li YY. Recent developments and advances of FGFR as a potential target in cancer. Future Med Chem. 2018;10(17):2109-26.

9. Dienstmann R, Rodon J, Prat A, Perez-Garcia J, Adamo B, Felip E, et al. Genomic aberrations in the FGFR pathway: opportunities for targeted therapies in solid tumors. Ann Oncol. 2014;25(3):552-63.

10. Weiss J, Sos ML, Seidel D, Peifer M, Zander T, Heuckmann JM, et al. Frequent and focal FGFR1 amplification associates with therapeutically tractable FGFR1 dependency in squamous cell lung cancer. Sci Transl Med. 2010;2(62):62-93.

11. Freier K, Schwaenen C, Sticht C, Flechtenmacher C, Muhling J, Hofele $\mathrm{C}$, et al. Recurrent FGFR1 amplification and high FGFR1 protein expression in oral squamous cell carcinoma (OSCC). Oral Oncol. 2007;43(1):60-6.

12. Elbauomy Elsheikh S, Green AR, Lambros MB, Turner NC, Grainge MJ, Powe D, et al. FGFR1 amplification in breast carcinomas: a chromogenic in situ hybridisation analysis. Breast Cancer Res. 2007;9(2):R23.

13. Theillet $\mathrm{C}$, Adelaide J, Louason G, Bonnet-Dorion F, Jacquemier $\mathrm{J}$, Adnane J, et al. FGFRI and PLAT genes and DNA amplification at $8 \mathrm{p} 12$ in breast and ovarian cancers. Genes Chromosomes Cancer. 1993;7(4):219-26.

14. Simon R, Richter J, Wagner U, Fijan A, Bruderer J, Schmid U, et al. High-throughput tissue microarray analysis of 3p25 (RAF1) and 8p12 (FGFR1) copy number alterations in urinary bladder cancer. Cancer Res. 2001;61(11):4514-9.

15. Lehnen NC, von Massenhausen A, Kalthoff H, Zhou H, Glowka T, Schutte U, et al. Fibroblast growth factor receptor 1 gene amplification in pancreatic ductal adenocarcinoma. Histopathology. 2013;63(2):157-66.

16. Yang Z, Klionsky DJ. Eaten alive: a history of macroautophagy. Nat Cell Biol. 2010;12(9):814-22. 
17. Yu L, Chen Y, Tooze SA. Autophagy pathway: cellular and molecular mechanisms. Autophagy. 2018;14(2):207-15.

18. Din FV, Valanciute A, Houde VP, Zibrova D, Green KA, Sakamoto K, et al. Aspirin inhibits mTOR signaling, activates AMPactivated protein kinase, and induces autophagy in colorectal cancer cells. Gastroenterology. 2012;142(7):1504-15 (e3).

19. Tsuchihara K, Fujii S, Esumi H. Autophagy and cancer: dynamism of the metabolism of tumor cells and tissues. Cancer Lett. 2009;278(2):130-8.

20. Ajibade AA, Wang HY, Wang RF. Cell type-specific function of TAK 1 in innate immune signaling. Trends Immunol. 2013;34(7):307-16.

21. Sakurai H. Targeting of TAK1 in inflammatory disorders and cancer. Trends Pharmacol Sci. 2012;33(10):522-30.

22. Seki E. TAK1-dependent autophagy: a suppressor of fatty liver disease and hepatic oncogenesis. Mol Cell Oncol. 2014;1(4):e968507.

23. Herrero-Martin G, Hoyer-Hansen M, Garcia-Garcia C, Fumarola C, Farkas T, Lopez-Rivas A, et al. TAK1 activates AMPKdependent cytoprotective autophagy in TRAIL-treated epithelial cells. EMBO J. 2009;28(6):677-85.

24. Inokuchi-Shimizu S, Park EJ, Roh YS, Yang L, Zhang B, Song $\mathrm{J}$, et al. TAK1-mediated autophagy and fatty acid oxidation prevent hepatosteatosis and tumorigenesis. J Clin Invest. 2014;124(8):3566-78.

25. Yuan K, Huang C, Fox J, Laturnus D, Carlson E, Zhang B, et al. Autophagy plays an essential role in the clearance of pseudomonas aeruginosa by alveolar macrophages. J Cell Sci. 2012;125(Pt 2):507-15.

26. Krishan S, Richardson DR, Sahni S. Gene of the month. AMP kinase (PRKAA1). J Clin Pathol. 2014;67(9):758-63.

27. Nakanishi Y, Mizuno H, Sase H, Fujii T, Sakata K, Akiyama N, et al. ERK signal suppression and sensitivity to CH5183284/ Debio 1347, a selective FGFR inhibitor. Mol Cancer Ther. 2015;14(12):2831-9.

28. Raoof S, Mulford IJ, Frisco-Cabanos H, Nangia V, Timonina D, Labrot E, et al. Targeting FGFR overcomes EMT-mediated resistance in EGFR mutant non-small cell lung cancer. Oncogene. 2019;38(37):6399-413.

29. Zhao Z, Wang L, Bartom E, Marshall S, Rendleman E, Ryan C, et al. Beta-catenin/Tcf712-dependent transcriptional regulation of GLUT1 gene expression by Zic family proteins in colon cancer. Sci Adv. 2019;5(7):eaax0698.

30. Liu W, Jiang Y, Sun J, Geng S, Pan Z, Prinz RA, et al. Activation of TGF-beta-activated kinase 1 (TAK1) restricts salmonella typhimurium growth by inducing AMPK activation and autophagy. Cell Death Dis. 2018;9(5):570.

31. Wong PM, Puente C, Ganley IG, Jiang X. The ULK1 complex: sensing nutrient signals for autophagy activation. Autophagy. 2013;9(2):124-37.

32. Alers S, Loffler AS, Wesselborg S, Stork B. Role of AMPKmTOR-Ulk1/2 in the regulation of autophagy: cross talk, shortcuts, and feedbacks. Mol Cell Biol. 2012;32(1):2-11.

33. Tripathi V, Shin JH, Stuelten $\mathrm{CH}$, Zhang YE. TGF-beta-induced alternative splicing of TAK1 promotes EMT and drug resistance. Oncogene. 2019;38(17):3185-200

34. Wu J, Du X, Li W, Zhou Y, Bai E, Kang Y, et al. A novel non-ATP competitive FGFR1 inhibitor with therapeutic potential on gastric cancer through inhibition of cell proliferation, survival and migration. Apoptosis. 2017;22(6):852-64.

35. Fischer KR, Durrans A, Lee S, Sheng J, Li F, Wong ST, et al. Epithelial-to-mesenchymal transition is not required for lung metastasis but contributes to chemoresistance. Nature. 2015;527(7579):472-6.

36. Zheng X, Carstens JL, Kim J, Scheible M, Kaye J, Sugimoto H, et al. Epithelial-to-mesenchymal transition is dispensable for metastasis but induces chemoresistance in pancreatic cancer. Nature. 2015;527(7579):525-30.

37. Kitai H, Ebi H, Tomida S, Floros KV, Kotani H, Adachi Y, et al. Epithelial-to-mesenchymal transition defines feedback activation of receptor tyrosine kinase signaling induced by MEK inhibition in KRAS-mutant lung cancer. Cancer Discov. 2016;6(7):754-69.

38. Gainor JF, Dardaei L, Yoda S, Friboulet L, Leshchiner I, Katayama R, et al. Molecular mechanisms of resistance to first- and second-generation ALK inhibitors in ALK-rearranged lung cancer. Cancer Discov. 2016;6(10):1118-33.

39. Amaravadi RK, Yu D, Lum JJ, Bui T, Christophorou MA, Evan GI, et al. Autophagy inhibition enhances therapy-induced apoptosis in a Myc-induced model of lymphoma. J Clin Invest. 2007;117(2):326-36.

40. Liu D, Yang Y, Liu Q, Wang J. Inhibition of autophagy by 3-MA potentiates cisplatin-induced apoptosis in esophageal squamous cell carcinoma cells. Med Oncol. 2011;28(1):105-11.

41. Apel A, Herr I, Schwarz H, Rodemann HP, Mayer A. Blocked autophagy sensitizes resistant carcinoma cells to radiation therapy. Cancer Res. 2008;68(5):1485-94.

42. Shingu T, Fujiwara K, Bogler O, Akiyama Y, Moritake K, Shinojima $\mathrm{N}$, et al. Inhibition of autophagy at a late stage enhances imatinib-induced cytotoxicity in human malignant glioma cells. Int J Cancer. 2009;124(5):1060-71.

43. Hu YL, Jahangiri A, Delay M, Aghi MK. Tumor cell autophagy as an adaptive response mediating resistance to treatments such as antiangiogenic therapy. Cancer Res. 2012;72(17):4294-9.

44. Dai L, Aye Thu C, Liu XY, Xi J, Cheung PC. TAK1, more than just innate immunity. IUBMB Life. 2012;64(10):825-34.

45. Neumann D. Is TAK1 a Direct Upstream Kinase of AMPK? Int J Mol Sci. 2018;19(8).

46. Papa S, Choy PM, Bubici C. The ERK and JNK pathways in the regulation of metabolic reprogramming. Oncogene. 2019;38(13):2223-40.

47. Yang W, Zheng Y, Xia Y, Ji H, Chen X, Guo F, et al ERK1/2-dependent phosphorylation and nuclear translocation of PKM2 promotes the Warburg effect. Nat Cell Biol. 2012;14(12):1295-304.

48. Hardie DG, Ross FA, Hawley SA. AMPK: a nutrient and energy sensor that maintains energy homeostasis. Nat Rev Mol Cell Biol. 2012;13(4):251-62.

Publisher's Note Springer Nature remains neutral with regard to jurisdictional claims in published maps and institutional affiliations. 


\section{Affiliations}

\section{Rui Peng ${ }^{1} \cdot$ Yan Chen ${ }^{2,5} \cdot{\text { Liangnian } \mathrm{Wei}^{3} \cdot \mathrm{Gang} \mathrm{Li}^{1} \cdot \text { Dongju Feng }^{3} \cdot \text { Siru Liu }^{2} \cdot \text { Runqiu Jiang }^{4} \cdot \text { Shaojiang Zheng }}^{2}$. Yun Chen ${ }^{1,3,6}$}

1 Department of General Surgery, Research Center for Clinical Oncology, The Affiliated Cancer Hospital of Nanjing Medical University, Jiangsu Cancer Hospital, Jiangsu Institute of Cancer Research, Nanjing 210009, Jiangsu, China

2 Key Laboratory of Emergency and Trauma of Ministry of Education, Tumor Institute of the First Affiliated Hospital, Hainan Medical University, Haikou 571199, China

3 Department of Immunology, Key Laboratory of Immune Microenvironment and Disease, Nanjing Medical University, Nanjing 211166, Jiangsu, China
4 Department of Hepatobiliary Surgery, The Affiliated Drum Tower Hospital of Nanjing University Medical School, Nanjing 210093, Jiangsu, China

5 Department of Ultrasound, Hainan General Hospital (Hainan Affiliated Hospital of Hainan Medical University), Haikou 570311, China

6 Jiangsu Key Lab of Cancer Biomarkers, Prevention and Treatment, Collaborative Innovation Center for Cancer Personalized Medicine, Nanjing Medical University, Nanjing 211166, Jiangsu, China 